\title{
Reply to: "Global conservation of phylogenetic diversity captures more than just functional diversity"
}

\author{
Florent Mazel (10) 1,2,3, Matthew W. Pennell ${ }^{3,4}$, Marc W. Cadotte 5,6, Sandra Diaz (1) 7, Giulio Valentino Dalla Riva ${ }^{8}$, \\ Richard Grenyer ${ }^{9}$, Fabien Leprieur ${ }^{10}$, Arne O. Mooers (1) ${ }^{1}$, David Mouillot ${ }^{10,11}$, Caroline M. Tucker ${ }^{12} \&$ \\ William D. Pearse (i) ${ }^{13}$
}

RePLying to N. R. Owen et al. Nature Communications https://doi.org/10.1038/s41467-019-08600-8 (2019)

\begin{abstract}
A cademic biologists have long advocated for conserving phylogenetic diversity (PD), often (but not exclusively) on the basis that PD is a useful proxy for "feature diversity", defined as the variety of forms and functions represented in set of organisms (see below for an extended discussion of this definition $)^{1-4}$. In a recent paper $^{5}$, we assess the extent to which this proxy (which we coined the "phylogenetic gambit") holds in three empirical datasets (terrestrial mammals, birds, and tropical marine fishes) when using functional traits and functional diversity (FD) to operationalize feature diversity. Owen et al. ${ }^{6}$ offer a criticism of our methods for quantifying feature diversity with FD and disagree with our conclusions. We are grateful that Owen et al. ${ }^{6}$ have engaged thoughtfully with our work, but we believe there are more points of agreement than Owen et al. ${ }^{6}$ imply.

The broad conclusion of our empirical study, which incorporated $>15,000$ vertebrate species and a limited number of traits, was that the phylogenetic gambit holds for ecologically relevant traits: maximizing PD results in an average gain of $18 \%$ of $\mathrm{FD}$ relative to random choice ${ }^{5}$. However, as clear from the title of our paper, PD's reliability as a surrogate for FD as we measured it was also surprisingly weak: the $18 \%$ gain masks the fact that, in $1 / 3$ of the comparisons, maximizing PD resulted in less FD than a random choice. This finding is in line with previous assessments 7,8 . Importantly, though, we did not argue that we "need to abandon the use of PD in conservation", and indeed
\end{abstract}

we opined that doing so would be a "dramatic decision"[5]. We called for further tests of the phylogenetic gambit in other taxonomic groups, at a variety of spatial scales, and across many types of traits.

Owen et al. ${ }^{6}$ offer three linked arguments: (1) FD is an inadequate measure of feature diversity in conservation; (2) our tests ignore spatial scale in conservation prioritization; and (3) our work is not supported by other tests of the phylogenetic gambit. We touch on each of these below.

(1) In the phylogenetic gambit, PD is a potential proxy for feature diversity. Feature diversity is an important concept, but as Faith" ${ }^{1}$ also noted, one that is "difficult to estimate directly". We provided one potential approach for operationalizing the concept, by measuring FD as is done in ecology. Importantly, we focused on ecological traits (body mass, diet, foraging strata, and foraging time) because we felt these are often related to conservation outputs of interest to humans (particularly via local ecosystem services). We emphasized that "many other potential[ly] valuable traits are not captured by our measure of $\mathrm{FD}^{\text {"5 }}$, but we do see how our phrase "diversity of form and function can be measured as FD" might be taken to include an implicit "exclusively." That was not our intent. Critically, there is some reason to believe that other measures of feature diversity (e.g., some integrated measures of total

\footnotetext{
${ }^{1}$ Department of Biological Sciences, Simon Fraser University, Burnaby V5A 156 BC, Canada. ${ }^{2}$ Department of Botany, University of British Columbia, Vancouver, BC V6T 1Z4, Canada. ${ }^{3}$ Biodiversity Research Centre, University of British Columbia, Vancouver, BC V6T 1Z4, Canada. ${ }^{4}$ Department of Zoology, University of British Columbia, Vancouver, BC V6T 1Z4, Canada. ${ }^{5}$ Biological Sciences, University of Toronto-Scarborough, Scarborough, M1C 1A4, Canada. ${ }^{6}$ Ecology and Evolutionary Biology, University of Toronto, Toronto M5S 3 B2 ON, Canada. ${ }^{7}$ Instituto Multidisciplinario de Biología Vegetal, CONICET and FECFyN, Universidad Nacional de Córdoba, Casilla de Correo 495, 5000 Córdoba, Argentina. ${ }^{8}$ School of Mathematics and Statistics, University of Canterbury, Christchurch 8140, New Zealand. ${ }^{9}$ School of Geography and the Environment, University of Oxford, Oxford OX1 3QY, UK. ${ }^{10}$ Marine Biodiversity, Exploitation, and Conservation (MARBEC), UMR 9190, Université de Montpellier, Montpellier 34095, France. ${ }^{11}$ Australian Research Council Centre of Excellence for Coral Reef Studies, James Cook University, Townsville 4811 QLD, Australia. ${ }^{12}$ Department of Biology, University of North Carolina-Chapel Hill, Chapel Hill 27599-3280 NC, USA. ${ }^{13}$ Ecology Center and Department of Biology, Utah State University, Logan 84322 UT, USA. Correspondence and requests for materials should be addressed to F.M. (email: flo.mazel@gmail.com)
} 
variation over many phenotypic axes) may covary differently with PD. This means that an essential next step will be to determine how trait choice, trait number and trait measurement impact the phylogenetic gambit. Critically, though, future operationalization of feature diversity must be coupled with tests of the potential benefits to people of what is being measured (see our point (3)). Thus, we fully agree with Owen et al. ${ }^{6}$ that our analysis is but one test of the phylogenetic gambit, and we hope this exchange spurs other approaches for measuring feature diversity.

(2) Owen et al. ${ }^{6}$ correctly highlight that our geographic (assemblage-based) prioritization analyses do not consider how PD captures FD at a global scale. Their empirical example of such global vs. local considerations for corals is useful. We did not consider corals but coral fishes in our paper, though they are mentioned in the newspaper article they quote. Our assemblage-based analysis, however, was only one half of our study: the other was a global analysis of the phylogenetic gambit in which we found results consistent with those of our assemblage-based analyses (surrogate estimates were $15 \%$ and $21 \%$, respectively). Thus, our results are likely robust to the concerns Owen et al. ${ }^{6}$ raise about our data and our measure of FD.

(3) While few would disagree with Owen et al. ${ }^{6}$ that their snake and marsupial anecdotes are exciting examples of the potential benefits of using phylogeny for prioritization and conservation, scientific hypotheses must be confronted with data, not anecdotes. Owen et al. ${ }^{6}$ cite a recent book chapter ${ }^{9}$ (ref. four) as a test of the phylogenetic gambit; however, we found only a single-empirical study referenced there. That study ${ }^{10}$ surveyed and found phylogenetic signal for various traits (although the pattern was not universal). We agree that phylogenetic signal is widespread and common ${ }^{11}$, but we have already shown ${ }^{8}$ that the presence of phylogenetic signal does not guarantee that the phylogenetic gambit holds as we tested it. This counter-intuitive result may be important as we consider further evidence.

Theory is foundational to scientific advance, but it is only a first step. We suggest that the only way to move this field forward is to empirically assess statistical support for all linkages between PD and benefits to people (see Forest et al. ${ }^{12}$ for one example). The issue is not academic: managers and policy makers grappling with urgent conservation decisions require evidence. We welcome further tests that link other metrics to other traits and to other explicit benefits.

Received: 18 October 2018 Accepted: 18 January 2019

Published online: 20 February 2019

\section{References}

1. Faith, D. P. Conservation evaluation and phylogenetic diversity. Biol. Conserv. 61, 1-10 (1992).

2. Winter, M., Devictor, V. \& Schweiger, O. Phylogenetic diversity and nature conservation: where are we? Trends Ecol. Evol. 28, 199-204 (2013).

3. Veron, S., Davies, T. J., Cadotte, M. W., Clergeau, P. \& Pavoine, S. Predicting loss of evolutionary history: where are we? Biol. Rev. 92, 271-291 (2017)
4. Rapacciuolo, G. et al. Species diversity as a surrogate for conservation of phylogenetic and functional diversity in terrestrial vertebrates across the Americas. Nat. Ecol. Evol. 3, 53-61 (2019).

5. Mazel, F. et al. Prioritizing phylogenetic diversity captures functional diversity unreliably. Nat. Commun. 9, 2888 (2018).

6. Owen, N. R., Gumbs, R., Gray, C. L. \& Faith, D. P. Matters arising: global conservation of phylogenetic diversity captures more than just functional diversity. Nat. Commun. https://doi.org/10.1038/s41467-019-08600-8 (2018).

7. Kelly, S., Grenyer, R. \& Scotland, R. W. Phylogenetic trees do not reliably predict feature diversity. Divers. Distrib. 20, 600-612 (2014). https://doi.org/ 10.1038/s41467-018-05126-3

8. Mazel, F., Mooers, A. O., Dalla Riva, G. V. \& Pennell, M. W. Conserving phylogenetic diversity can be a poor strategy for conserving functional diversity. Syst. Biol. 66, 1019-1027 (2017).

9. Faith, D. P. Phylogenetic diversity and conservation evaluation: perspectives on multiple values, indices, and scales of application. In Phylogenetic Diversity. Applications and Challenges in Biodiversity Science (eds Scherson, R. \& Faith, D. P.) (Springer, New York, 2018).

10. Slowinski, J. \& Crother, B. I. Is the PTP test useful? Cladistics 14, 297-302 (1998).

11. Blomberg, S. P., Garland, T. J. R. \& Ives, A. Testing for phylogenetic signal in comparative data: behavioral traits are more labile. Evolution 57, 717-745 (2003).

12. Forest, F. et al. Preserving the evolutionary potential of floras in biodiversity hotspots. Nature 445, 757-760 (2007).

\section{Acknowledgments}

This paper is a joint effort of the sCAP working group funded by sDIV, the Synthesis Centre of the German Centre for Integrative Biodiversity Research Halle-Jena-Leipzig and the Canadian Institute for Ecology and Evolution. FM was supported by an NSERC Accelerator Grant to AOM and a Banting Post-doctoral fellowship. GDR thanks Mike Steel. We thank Risa Sergeant at the University of Ottawa for hospitality, and the Crawford Lab for Evolutionary Studies at SFU for discussion. M.C., D.M., and C.T. are members of the FREE working group at the CESAB-FRB.

\section{Author contributions}

F.M. and W.D.P. drafted the first version of the manuscript. F.L., D.M., M.W.P., M.W.C. S.D., G.V.D.R., R.G., A.O.M., and C.M.T. provided feedback. F.M. and A.O.M. edited the final version.

\section{Additional information}

Competing interests: The authors declare no competing interests.

Reprints and permission information is available online at http://npg.nature.com/ reprintsandpermissions/

Journal peer review information: Nature Communications thanks the anonymous reviewers for their contribution to the peer review of this work.

Publisher's note: Springer Nature remains neutral with regard to jurisdictional claims in published maps and institutional affiliations.

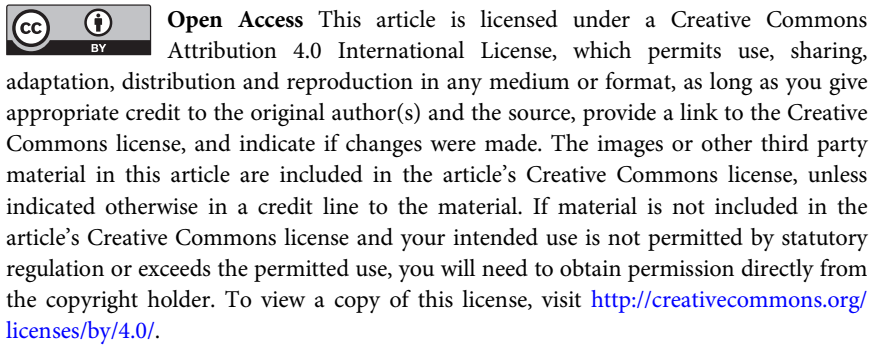
licenses/by/4.0/.

(c) The Author(s) 2019 